Pacific Journal of Mathematics

ALGEBRAS OF GLOBAL DIMENSION ONE WITH A FINITE

WIUUA EDwin CLARK 


\title{
ALGEBRAS OF GLOBAL DIMENSION ONE WITH A FINITE IDEAL LATTICE
}

\author{
W. EDWIN CLARK
}

Let $A$ denote a finite-dimensional (associative) algebra over an algebraically closed field $K$. It is well known that $A$ has global dimension zero if and only if $A$ is the direct sum of a finite number of full matrix algebras over $K$. In this paper a specific representation is given for those algebras $A$ which have global dimension one (or less) and have only a finite number of (two-sided) ideals. It is shown that every such algebra is isomorphic to a (contracted) semigroup algebra $K[S]$ over a subsemigroup $S$ of the semigroup of all $n \times n$ matrix units $\left\{e_{i j}\right\} \cup\{0\}$ which (i) contains $e_{11}, \cdots, e_{n n}$ and (ii) contains $e_{i j}$ or $e_{j i}$ whenever there are $h$ and $k$ such that $e_{h i}, e_{i k}$ and $e_{h j}, e_{j k}$ are in $S$. Conversely, if $S$ satisfies (i) and (ii) then $K[S]$ has global dimension one or less and has a finite ideal lattice.

We use the definitions and notation of Cartan-Eilenberg ([2], VI, 2) and Jans ([11],4). If $A$ is a finite-dimensional algebra then $A$ is Noetherian and therefore l.gl. $\operatorname{dim} . A=\mathrm{r} . \mathrm{gl} . \operatorname{dim} . A$. In this case one writes gl. $\operatorname{dim} . A$ for this number. It is perhaps worthwhile to point out that if $A$ is over an algebraically closed field, then gl.dim. $A$ is precisely dim. $A$, the so-called Hochschild dimension of $A$ (see [2], p. 176) and [8]). In [10] Hochschild proved that $\operatorname{dim} . A \leqq 1$ if and only if $A$ is segregated in every extension, i.e., every exact sequence of (finite-dimensional) algebras $B \rightarrow A \rightarrow 0$ splits. In [12] Jans gives a structure theorem for this class of algebras. By the above comments, for algebraically closed fields Jans' theorem is in fact a structure theorem for algebras of global dimension one or less. Unfortunately, however, we are unable at this time to relate the results of this paper to those of Jans.

Harada [9] has also given a characterization of semiprimary rings of global dimension $\leqq 1$ which is in spirit somewhat related to the methods of this paper. But again we are unable to deduce our results from Harada's.

On the other hand, Barry Mitchell has pointed out to the author that part of the main theorem of this paper is an immediate corollary of his work on the global dimension of abelian categories, see [15], pp. $229 \mathrm{ff}$. Specifically, one infers immediately from Mitchell's results that if $S$ is a subsemigroup of $\left\{e_{i j}\right\} \cup\{0\}$ which contains all $e_{i i}$, then gl. $\operatorname{dim} . K[S] \leqq 1$ if and only if whenever $e_{h i}, e_{i k}$ and $e_{h j}, e_{j k}$ are in $S$ then either $e_{i j}$ or $e_{j i}$ is also in $S$. In this paper, however, we prefer 
to retain our original proofs since they require no special knowledge of category theory.

For convenience we define a semigroup $S$ of matrix units (of degree $n$ ) to be a subsemigroup of the semigroup of all $n \times n$ matrix units $\left\{e_{i j}\right\} \cup\{0\}$ which contains all $e_{i i}$. If $K$ is a field, $K[S]$ will denote the algebra of all $n \times n$ matrices over $K$ which is spanned by $S$. If $n=1$ and $S=\left\{e_{11}\right\}$, then $K[S] \cong K$ is the semigroup algebra of $S$ over $K$; in all other cases $S$ contains $e_{11}$ and $e_{22}$ and therefore contains $0=e_{11} e_{22}$. In this case $K[S]$ is the so-called contracted semigroup algebra of $S$ over $K$, i.e., the semigroup algebra of $S$ over $K$ modulo the ideal generated by the zero of $S$ (cf. [6]).

In general $K[S]$ has global dimension greater than one. The smallest $K[S]$ which is not of global dimension $\leqq 1$ is the algebra of all $4 \times 4$ matrices

$$
\left(\begin{array}{cccc}
x_{11} & 0 & 0 & 0 \\
x_{21} & x_{22} & 0 & 0 \\
x_{31} & 0 & x_{33} & 0 \\
x_{41} & x_{42} & x_{43} & x_{44}
\end{array}\right), x_{i j} \text { in } K .
$$

Ignoring the zeros above the main diagonal, we note that the zero in the $(3,2)$-position is "surrounded by nonzero positions". To describe this situation more precisely we introduce the graph

$$
G(S)=\left\{(i, j): e_{i j} \in S\right\}
$$

of a matrix units semigroup $S$. Clearly there is a one-one correspondence between transitive, reflexive (directed) graphs on $n$ vertices and matrix units semigroups of degree $n$. We say that $S$ (or $G(S)$ ) surrounds no zeros if whenever $(h, i),(i, k)$ and $(h, j),(j, k)$ are elements of $G(S)$, then either $(i, j)$ or $(j, i)$ is also in $S$. This is equivalent to the existence of unique paths of maximal length joining any two vertices. Mitchell ([15], p. 236) calls such a graph a decision free graph.

We now state our main result:

THEOREM. Let $A$ be a finite-dimensional algebra with identity over an algebraically closed field $K$. Then $A \cong K[S]$ for some semigroup $S$ of matrix units which surrounds no zeros if and only if gl. dim. $A \leqq 1$ and $A$ has only a finite number of ideals.

The remainder of the paper will be devoted to a proof of this theorem.

Lemma 1. If $A=K[S]$ where $S$ is a semigroup of matrix units 
which surrounds no zeros, then $\mathrm{gl} \cdot \operatorname{dim} A \leqq 1$.

Proof. Let $e_{i}=e_{i i}$, and let $N$ denote the radical of $A$. Since $1=\Sigma e_{i}$ and the $e_{i}$ are primitive, each simple $A$-module has the form $A e_{i} / N e_{i}$, [7]. It therefore suffices by ([11], p. 56) to show that $N e_{i}$ is projective for each $i$.

As in the introduction to [4], we may assume that the vertex incidence matrix $C=\left(c_{i j}\right)\left(c_{i j}=0\right.$ if $e_{i j} \notin S$ and $c_{i j}=1$ if $\left.e_{i j} \notin S\right)$ has the block triangular form

$$
\left(\begin{array}{cccccc}
C_{11} & & & & & \\
C_{21} & C_{22} & & & & 0 \\
\cdot & \cdot & \cdot & & & \\
\cdot & \cdot & & \cdot & & \\
\cdot & \cdot & & & \cdot & \\
C_{m 1} & C_{m 2} & \cdot & \cdot & \cdot & C_{m m}
\end{array}\right)
$$

where the diagonal blocks are square matrices each entry of which is 1 and each of the blocks below the diagonal is either a zero block or else has all entries equal to 1 .

Let $J$ be the set of $i$ such that $e_{i p} \in N e_{p}$. Clearly, $N e_{p}$ is the vector space direct sum of the $K e_{i p}, i \in J$. Let $G=G(S)$. Set $n_{1}=$ $\min J$ and define

$$
J_{1}=\left\{i:\left(i, n_{1}\right) \in G\right\} \text {. }
$$

Since $n_{1} \in J,\left(n_{1}, p\right) \in G$; hence for $i \in J_{1}$ we have $e_{i p}=e_{i n_{1}} e_{n_{1} p} \in N e_{p}$. Thus $J_{1} \subseteq J$.

Having defined integers $n_{1}<n_{2}<\cdots<n_{k}$ in $J$ and subsets $J_{1}, \cdots, J_{k}$ of $J$ such that for each $t \leqq k n_{t}=\min J-\left(J_{1} \cup \cdots \cup J_{t-1}\right)$ and $J_{t}=\left\{i:\left(i, n_{t}\right) \in G\right\}$, if $J \neq J_{1} \cup \cdots \cup J_{k}$, let us define

$$
n_{k+1}=\min J-\left(J_{1} \cup \cdots \cup J_{k}\right)
$$

and $J_{k+1}=\left\{i:\left(i, n_{k+1}\right) \in G\right\}$. As in the case above for $n_{1}$ we have $J_{k+1} \subseteq J$. By the way we chose $n_{t}$ for $t \leqq k$, clearly $n_{k+1}>n_{k}$. Since $J$ is finite this process must end, and so, there is an integer $m$ such that $J=J_{1} \cup \cdots \cup J_{m}$.

We now show that the $J_{i}$ are pair-wise disjoint. Assume that for $s>t, J_{s} \cap J_{t}$ contains a nonzero element $i$. Then $\left(i, n_{s}\right)$ and $\left(i, n_{t}\right) \in G$. Since $n_{s}, n_{t} \in J$ we also have $\left(n_{s}, p\right)$ and $\left(n_{t}, p\right)$ in $G$. But, $\left(n_{s}, n_{t}\right) \notin G$ since $s>t$ implies that $n_{s} \notin J_{t}$. Now since $S$ surrounds no zeros we must have $\left(n_{t}, n_{s}\right)$ in $G$. This implies that the vertex incidence matrix $C$ has a diagonal block (not necessarily one of the $C_{i i}$ ) of the form: 


$$
\left(\begin{array}{ccc}
1 & \cdots & 1 \\
\vdots & & \vdots \\
0 & \cdots & 1
\end{array}\right)
$$

where the three ones correspond to the edges $\left(n_{t}, n_{t}\right),\left(n_{t}, n_{s}\right),\left(n_{s}, n_{s}\right)$ and the zero represents the fact that $\left(n_{s}, n_{t}\right) \notin G$. But this contradicts the fact that $C$ has square diagonal blocks with all entries 1 . Hence we have $J_{s} \cap J_{t}=\varnothing$.

Let now $M_{i}=\Sigma\left\{K e_{j p}: j \in J_{i}\right\}$. We claim that $M_{i}$ is a left ideal of $A$. To show this it suffices to show that if $e_{k j} \in S$ where $j \in J_{i}$, then $k \in J_{i}$ : Note that $j \in J_{i}$ if and only if $\left(j, n_{i}\right) \in G$; hence if $(k, j) \in G$ and $j \in J_{i}$, by transitivity $\left(k, n_{i}\right) \in G$ and so $k \in J_{i}$. Since the $J_{i}$ are pairwise disjoint we have $N e_{p}=M_{1} \oplus \cdots \oplus M_{m}$.

Observe next that $A e_{n_{i}}=\Sigma\left\{K e_{j_{i}}: j \in J_{i}\right\}$. Now one easily verifies that the mapping $\varphi: M_{i} \rightarrow A e_{n_{i}}$ defined by $\varphi\left(\Sigma \alpha_{j} e_{j p}\right)=\Sigma \alpha_{j} e_{j n_{i}}$ is an $A$ isomorphism. It follows that $N e_{p}$ is isomorphic to the direct sum of the projective $A$-modules $A e_{n_{i}}$, and is therefore itself projective. This completes the proof of the lemma.

One easily verifies that every ideal $I$ of a matrix units semigroup algebra $K[S]$ is generated by $I \cap S$ and so $K[S]$ has only a finite number of ideals. This fact with Lemma 1 proves one half of our theorem.

Recall that a ring $R$ is called hereditary if every left ideal is projective. It is well known that $l$.gl. dim. $R \leqq 1$ if and only if $R$ is hereditary (see [2], p. 112). If $X \subseteq R$, let $l(X)$ denote the left annihilator of $X$ in $R$. After Kaplansky [13], we call a ring Baer if it has an identity and the left annihilator of every subset is generated by an idempotent.

Lemma 2. A hereditary, Artinian ring (with identity) is a Baer ring.

Proof. If $a \in R$, then, $R a$ is a left ideal of $R$ and therefore projective. Hence $R \stackrel{\varphi}{\longrightarrow} R a \rightarrow 0$ splits where $\varphi(r)=r a$. This says that $\operatorname{ker} \varphi=l(a)$ is a direct summand of ${ }_{R} R$. If ${ }_{R} R=L \oplus l(a), 1=f+e$ where $f \in L, e \in L, e \in l(a)$, then $l(a)=R e$ and $e^{2}=e$.

By an argument due to Maeda [14] (which we include for the convenience of the reader) we can extend this to two elements: Let $a, b \in R$. If $l(a)=R e$ and $l(b)=R f$ where $e^{2}=e, f^{2}=f$, then $l(a)=$ $l(1-e)$ and $l(b)=l(1-f)$. As shown above there is an idempotent $g$ such that $l(e(1-f))=R g$. It is now straightforward to show that $(g e)^{2}=g e$ and that $l(a, b)=l(1-e, 1-f)=R g e$.

Now since $R$ is Artinian one show easily that $l(X)=A e$ for any subset $X$ of $R$. 
DeFinition. Let $S$ be a semigroup of matrix units. By a twisted matrix units semigroup algebra of $S$ over a field $K$ we shall mean an algebra $K_{\varphi}[S]$ which has a basis $\left\{a_{i j}:(i, j) \in G(S)\right\}$ which multiplies as follows: $a_{i j} a_{j k}=\varphi(i, j, k) a_{i k}$ where $\varphi(i, j, k)$ is a nonzero element of $K$; all other products are zero.

In case $\varphi=1$ (when defined), we clearly have that $K_{\varphi}[S] \cong K[S]$ where $K[S]$ is the (contracted) semigroup algebra of $S$ over $K$.

The following lemma follows immediately from results in [5], however, for the sake of completeness we give a proof here.

LEMмA 3. Let $A$ be a finite dimensional algebra over an algebraically closed field $K$. If $A$ is Baer and has a finite ideal lattice then $A$ is a twisted matrix units semigroup algebra over $K$.

Proof. First we note that if $A$ is Baer and if $e$ is an idempotent in $A$, then $e A e$ is Baer (see [14]). Hence, if $e$ is primitive then $e A e$ has only one idempotent and therefore the left annihilator of every nonzero subset is zero. This implies that the radical of $e A e$ is zero and that $e A e$ is a divisor ring. Since $K$ is algebraically closed we have then that $e A e=K e$.

Now let $1=\Sigma e_{i}$ where $\left\{e_{i}\right\}$ is a family of pairwise orthogonal idempotents. Let us first show that if $e_{i} x e_{j} y e_{k}=0$, then $e_{i} x e_{j}=0$ or $e_{i} y e_{k}=0$ : Suppose $e_{j} y e_{k} \neq 0$ and let $A f, f^{2}=f$, be the left annihilator of $e_{j} y e_{k}$. Now $f e_{j} y e_{k}=0$ and hence $e_{j} f e_{j} y e_{k}=0$; since $e_{j} f e_{j} \in K e_{j}$ we must have $e_{j} f e_{j}=0$. On the other hand, $e_{i} x e_{j} \in A f$ and so $e_{i} x e_{j}=$ $e_{i} x e_{j} f$. Multiplying on the right by $e_{j}$ we obtain $e_{i} x e_{j}=0$.

Now it is clear that to complete the proof of this lemma it suffices to show that $e_{i} A e_{j}$ has dimension $\leqq 1$ over $K$ for all $i, j$. Since $e A e$ has finite ideal lattice for all $e^{2}=e$, it suffices to assume that $1=$ $e_{1}+e_{2}$. First suppose that $e_{1} A e_{2} \neq 0$ and $e_{2} A e_{1} \neq 0$. Then, as shown above, $e_{2} A e_{1} A e_{2} \neq 0$ and therefore $e_{2} A e_{1} A e_{2}=e_{2} A e_{2}$. Hence, there exist $e_{21} \in e_{2} A e_{1}$ and $e_{12} \in e_{1} A e_{2}$ such that $e_{21} e_{12}=e_{2}$. Now, if $x_{21} \in e_{2} A e_{1}$ we have $x_{21}=e_{2} x_{21}=e_{21} e_{12} x_{21}=\mathrm{e}_{21}\left(\alpha e_{1}\right)=\alpha e_{21}$. Thus, $\left[e_{2} A e_{1}: K\right]=1$. Similarly, $\left[e_{1} A e_{2}: K\right]=1$. In the remaining case assume that $e_{1} A e_{2}=0$. It follows that $A=K e_{1}+K e_{2}+e_{2} A e_{1}$. One easily shows that any $K$-subspace of $e_{2} A e_{1}$ is an ideal of $A$. Since $K$ is infinite and $A$ has only finitely many ideals we can only conclude that $\left[e_{2} A e_{1}: K\right] \leqq 1$.

Lemma 3 together with Lemma 2 tells us that a finite-dimensional, hereditary algebra with finite ideal lattice over an algebraically closed field $K$ is isomorphic to a twisted matrix units semigroup algebra $K_{\varphi}[S]$. Thus, to complete the proof of our main result we need only show that such an $S$ surrounds no zeros and that $K_{\varphi}[S] \cong K[S]$.

LEMMA 4. Let $S$ be a semigroup of matrix units such that 
$A=K_{\varphi}[S]$ is Baer. Then, $S$ surrounds no zeros.

Proof. Let $a_{p q},(p, q) \in G=G(S)$ be a basis for $A$ satisfying the conditions in the above definition. Clearly we may choose $a_{p p}$ to be idempotent. We write $e_{p}=a_{p p}$.

Now suppose that $S$ does surround a zero. Then there exist $(h, i),(i, k),(h, j),(j, k) \in G$, with $(i, j)$ and $(j, i)$ not in $G$. Let $e=$ $e_{h}+e_{i}+e_{j}+e_{k}$. Since $A$ is Baer, $e A e$ is also (see [13]). But $e A e$ is not Baer. To see this observe that $e A e$ has a basis consisting exactly of the elements of the array:

$$
\begin{array}{llll}
a_{k k} & & & \\
a_{i k} & a_{i i} & & \\
a_{j k} & & a_{j j} & \\
a_{h k} & a_{h i} & a_{h j} & a_{h h} .
\end{array}
$$

Note that $(p, q) \in G$ if and only if $e_{p} A e_{q} \neq 0$. Now suppose that $e_{k} A e_{i} \neq 0$, then $a_{k i}$ is a basis element of $A$. But then

$$
0 \neq a_{j k} a_{k i} \in e_{j} A e_{i} .
$$

Hence $(j, i) \in G$, contrary to our assumption. Similar arguments show that the basis elements $a_{p q}$ in the above array are indeed the only ones which survive in $e A e$. It follows easily that $e A e$ is isomorphic to the algebra of all $4 \times 4$ matrices

$$
x=\left(\begin{array}{cccc}
x_{11} & 0 & 0 & 0 \\
x_{21} & x_{22} & 0 & 0 \\
x_{31} & 0 & x_{33} & 0 \\
x_{41} & x_{42} & x_{43} & x_{44}
\end{array}\right)
$$

where $x_{i j} \in K$. But the left annihilator of the element $x$ where $x_{21}=x_{31}=1$ and all other entries are zero is not generated by an idempotent. This establishes the lemma.

LEMMA 5. Let $S$ be a semigroup of matrix units which surrounds no zeros. Then, $K_{\varphi}[S] \cong K[S]$.

Proof. Let $A=K_{\varphi}[S]$. As in the proof of Lemma 1, we assume that the vertex incidence matrix $C$ of the graph $G$ of $S$ has the normalized form (1). If $C_{i i}$ is an $n_{i} \times n_{i}$ block, then $A / \operatorname{rad} A$ is isomorphic to the direct sum of algebras $K_{\varphi_{i}}\left[T_{i}\right]$ where $T_{i}$ is the semigroup of all matrix units of degree $n_{i}$. One shows easily that

$$
K_{\varphi_{i}}\left[T_{i}\right] \cong K\left[T_{i}\right]
$$

and hence that 


\section{$A / \operatorname{rad} A \cong K[S] / \operatorname{rad} K[S]$}

We conclude from this that if the reduced (basic) ring of $A$ is isomorphic to that of $K[S]$, then $A$ is isomorphic to $K[S]$ (see [1]).

Now from the block triangular form of $C$, it is clear that the reduced ring of $A$ is of the form $K_{\varphi}\left(S^{\prime}\right)$ where $S^{\prime}$ is a semigroup of matrix units whose associated graph is strictly triangular (not just block triangular). Thus, without loss of generality we may assume that $S=S^{\prime}$ and, hence that $(i, j) \in G(S)$ implies that $j \leqq i$.

We now show that if $\left\{a_{i j}:(i, j) \in G=G(S)\right\}$ is a basis for $A$ (which satisfies the conditions in the above definition) then each $a_{i j}$ may be replaced by a nonzero $K$-multiple $a_{i j}^{\prime}$ of $a_{i j}$ so that the basis

$$
\left\{a_{i j}^{\prime}:(i, j) \in G\right\}
$$

together with zero is a semigroup (necessarily isomorphic to $S$ ).

First we choose $a_{i i}^{\prime}$ so that $a_{i i}^{\prime}$ is idempotent. Clearly this can be done since $a_{i i}^{2}=\alpha(i, i, i) a_{i i}$ and $\alpha(i, i, i) \neq 0$. Now replace $a_{i j}$ by $a_{i i}^{\prime} a_{i j} a_{j j}^{\prime}$, so that without loss of generality we may assume that $a_{i i}^{2}=a_{i i}$ and $a_{i i} a_{i j}=a_{i j} a_{j j}=a_{i j}$.

Let now $m$ denote the degree of $S$ and let $n<m$. Assume inductively that we have replaced all $a_{i j}$ for $i<n$ by nonzero $K$ multiples $a_{i j}^{\prime}$ so that the set $T$ of all $a_{i j}^{\prime},(i, j) \in G$ and $i<n$, together with zero is a semigroup. Let the " $n$-th row" of $S$ consist of

$$
a_{n i_{p}}, \cdots, a_{n i_{1}}, a_{n n} \text {. }
$$

Assume that we have replaced the last $s+1$ elements of this row by $a_{n i_{s}}^{\prime}, \cdots, a_{n i_{1}}^{\prime}, a_{n n}^{\prime}=a_{n n}$, so that $T_{s}=T \cup\left\{a_{n i_{s}}^{\prime}, \cdots, a_{n i_{1}}^{\prime}, a_{n n}^{\prime}\right\}$ satisfies the following condition:

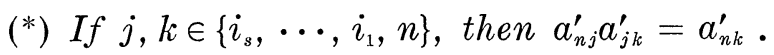

Choose $a_{n i_{s+1}}^{\prime}=a_{n t}^{\prime} a_{t i_{s+1}}^{\prime}$, if there exists $t \in\left\{i_{s}, \cdots, i_{1}\right\}$ such that $\left(t, i_{s+1}\right) \in G$. If there is no such $t$, let $a_{n i_{s+1}}^{\prime}=a_{n i_{s+1}}$. We now claim that $T_{s+1}$ satisfies (*) with $s+1$ replacing $s:$ Since $(j, k) \in G$ implies that $k \leqq j$, it suffices to show that for any $j$ such that $n>j>i_{s+1}$ and $\left(j, i_{s+1}\right) \in G$ we have $a_{n j}^{\prime} a_{j i_{s+1}}^{\prime}=a_{n t}^{\prime} a_{t i_{s+1}}^{\prime}$ : (The case where $a_{n i_{s+1}}^{\prime}=a_{n i_{s+1}}$ is clear). This means that $(n, j),\left(j, i_{s+1}\right)$ and $(n, t),\left(t, i_{s+1}\right)$ are in $G$. Since $S$ surrounds no zeros we must therefore have $(j, t)$ or $(t, j)$ in $G$. Assume that $(t, j) \in G$ then $a_{t i_{s+1}}^{\prime}=a_{t j}^{\prime} a_{j i_{s+1}}^{\prime}$ and hence by our inductive hypotheses $a_{n t}^{\prime} a_{t_{i s+1}}^{\prime}=a_{n t}^{\prime} a_{t j}^{\prime} a_{j i_{s+1}}^{\prime}=a_{n j}^{\prime} a_{j i_{s+1}}^{\prime}$. A similar argument takes care of the case $(j, t) \in G$. This shows that $T_{s+1}$ satisfies (*). By induction $T_{p}$ satisfies (*) with $s=p$, i.e., $T_{p}$ together with zero in a semigroup. Now it is clear that by induction on $n$, we can choose $\left\{a_{i j}^{\prime}\right\}$ as desired. This completes the proof of the lemma and therefore of the theorem. 
REMARKs. Examples show that the situation gets much more complicated if one weakens any of the hypotheses of our main theorem. On the other hand, the only place that the algebraic closure of $K$ is needed is in Lemma 3. That it is essential there is shown by the real algebra of all $2 \times 2$ matrices of the form

$$
\left(\begin{array}{ll}
z_{1} & 0 \\
z_{2} & t
\end{array}\right)
$$

where $z_{i}$ are complex and $t$ is real.

A partial generalization in one direction may be obtained as follows: Let $A$ be finite-dimensional over an algebraically closed field $K$. Instead of assuming that $A$ is hereditary assume the weaker condition that gl. $\operatorname{dim} . A / N^{2}<\infty$. Stephen Chase [3] has shown that this is equivalent to the existence of a complete set of mutually orthogonal idempotents $e_{1}, \cdots, e_{n}$ such that $e_{i} N e_{j}=0$ if $i \geqq j$. Now, if we assume further that $A$ has a finite ideal lattice, a slight extension of the argument in the proof of Lemma 3 (above) shows that $\left[e_{i} A e_{j}: K \mid \leqq 1\right.$ for all $i, j$. Thus, $A$ has a basis $t_{i j}=e_{i} t_{i j} e_{j}$ such that

$$
t_{i j} t_{j k}=\varphi(i, j, k) t_{i k}
$$

where $\varphi(i, j, k)$ is some element (possibly zero) of $K$. However, even if we assume that $\varphi(i, j, k) \neq 0$ (when defined) it is in general impossible to replace $\varphi(i, j, k)$ by 1 and get an isomorphic algebra (see the example in [5]). It is, of course, quite possible that one might be able to find reasonable necessary and sufficient conditions on the graph $G=\left\{(i, j): e_{i} A e_{j} \neq 0\right\}$ and the function $\varphi$ in order that $A$ have global dimension $\leqq n$.

We wish also to point out that Lemma 1 together with Lemma 2 yields a new and somewhat less complicated (modulo basic results on the global dimension of Artinian rings) proof of our theorem in 14$]$ that for a divisor ring $K, K[S]$ is a Baer ring if $S$ is a semigroup of matrix units which surrounds no zeros.

\section{REFERENCES}

1. R. Brauer, Some remarks on associative rings and algebras, National Acad. of Sciences-National Research Council, Publication 502, Washington, 1957.

2. H. Cartan and S. Eilenberg, Homological Algebra, Pxinceton University Press, Princeton, 1956.

3. S. U. Chase, A generalization of the ring of triangular matrices, Nagoya Math. J. 18 (1961), 13-25.

4. W. E. Clark, Baer rings which arise from certain transitive graphs, Duke Math.

J. Dec. (1966), 647-656.

5. —, Twisted matrix units semigroup algebras (to appear in Duke Math. J.)

6. A. H. Clifford and G. B. Preston, The algebraic theory of semigroups, Vol. I, Math. Surveys, No. 7, Amer. Math. Soc., 1961. 
7. C. W. Curtis and I. Reiner, Representation Theory of Finite Groups and Associative Algebras, Interscience Pub., New York, 1962.

8. S. Eilenberg, Algebras of cohomologically finite dimension, Comment. Math. Helv. 28 (1954), 310-319.

9. M. Harada, Hereditary semi-primary rings and triangular matrix rings, Nagoya Math. J. 27 (1966), 463-484.

10. G. Hochschild, On the cohomology of an associative algebra, Ann. of Math. 46 (1945), 58-67.

11. J. P. Jans, Rings and Homology, Holt, New York, 1964.

12. - On segregated rings and algebras, Nagoya Math. J. 11 (1957), 65-71.

13. I. Kaplansky, Rings of operators, Univ. of Chicago mimeographed notes, 1955.

14. S. Maeda, On a rings whose principal right ideals generated by idempotents form a lattice, Jour. of Sc. Hiroshima Univ. (1960), 509-525.

15. B. Mitchell, Theory of Categories, Academic Press, New York, 1965.

Received March 31, 1967.

UNIVERSITY OF FLORIDA 



\section{PACIFIC JOURNAL OF MATHEMATICS}

\section{H. ROYDEN}

Stanford University

Stanford, California

\author{
J. P. JANS \\ University of Washington \\ Seattle, Washington 98105
}

\section{EDITORS}

\author{
J. DugundJI \\ Department of Mathematics \\ Rice University \\ Houston, Texas 77001 \\ Richard ARENS \\ University of California \\ Los Angeles, California 90024
}

\section{ASSOCIATE EDITORS}
E. F. BECKENBACH
B. H. NeumanN
F. WOLF
K. YOSIDA

\section{SUPPORTING INSTITUTIONS}

\author{
UNIVERSITY OF BRITISH COLUMBIA \\ CALIFORNIA INSTITUTE OF TECHNOLOGY \\ UNIVERSITY OF CALIFORNIA \\ MONTANA STATE UNIVERSITY \\ UNIVERSITY OF NEVADA \\ NEW MEXICO STATE UNIVERSITY \\ OREGON STATE UNIVERSITY \\ UNIVERSITY OF OREGON \\ OSAKA UNIVERSITY \\ UNIVERSITY OF SOUTHERN CALIFORNIA
}

\author{
STANFORD UNIVERSITY \\ UNIVERSITY OF TOKYO \\ UNIVERSITY OF UTAH \\ WASHINGTON STATE UNIVERSITY \\ UNIVERSITY OF WASHINGTON \\ AMERICAN MATHEMATICAL SOCIETY \\ CHEVRON RESEARCH CORPORATION \\ TRW SYSTEMS \\ NAVAL ORDNANCE TEST STATION
}

Mathematical papers intended for publication in the Pacific Journal of Mathematics should be typewritten (double spaced). The first paragraph or two must be capable of being used separately as a synopsis of the entire paper. It should not contain references to the bibliography. Manuscripts may be sent to any one of the four editors. All other communications to the editors should be addressed to the managing editor, Richard Arens at the University of California, Los Angeles, California 90024.

50 reprints per author of each article are furnished free of charge; additional copies may be obtained at cost in multiples of 50 .

The Pacific Journal of Mathematics is published monthly. Effective with Volume 16 the price per volume (3 numbers) is $\$ 8.00$; single issues, $\$ 3.00$. Special price for current issues to individual faculty members of supporting institutions and to individual members of the American Mathematical Society: $\$ 4.00$ per volume; single issues $\$ 1.50$. Back numbers are available.

Subscriptions, orders for back numbers, and changes of address should be sent to Pacific Journal of Mathematics, 103 Highland Boulevard, Berkeley 8, California.

Printed at Kokusai Bunken Insatsusha (International Academic Printing Co., Ltd.), 7-17, Fujimi 2-chome, Chiyoda-ku, Tokyo, Japan.

PUBLISHED BY PACIFIC JOURNAL OF MATHEMATICS, A NON-PROFIT CORPORATION

The Supporting Institutions listed above contribute to the cost of publication of this Journal, but they are not owners or publishers and have no responsibility for its content or policies. 


\section{Pacific Journal of Mathematics}

A. A. Aucoin, Diophantine systems ............................. 419

Charles Ballantine, Products of positive definite matrices. I ............... 427

David Wilmot Barnette, A necessary condition for d-polyhedrality ............ 435

James Clark Beidleman and Tae Kun Seo, Generalized Frattini subgroups of finite groups ......................................... 441

Carlos Jorge Do Rego Borges, A study of multivalued functions ............. 451

William Edwin Clark, Algebras of global dimension one with a finite ideal

lattice ...............................................

Richard Brian Darst, On a theorem of Nikodym with applications to weak convergence and von Neumann algebras .........................

George Wesley Day, Superatomic Boolean algebras .....................

Lawrence Fearnley, Characterization of the continuous images of all

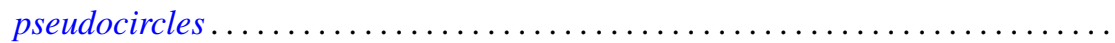

Neil Robert Gray, Unstable points in the hyperspace of connected subsets....... 515

Franklin Haimo, Polynomials in central endomorphisms .................. 521

John Sollion Hsia, Integral equivalence of vectors over local modular lattices . . . . 527

Jim Humphreys, Existence of Levi factors in certain algebraic groups .......... 543

E. Christopher Lance, Automorphisms of postliminal $C^{*}$-algebras ............ 547

Sibe Mardesic, Images of ordered compacta are locally peripherally metric . . . . 557

Albert W. Marshall, David William Walkup and Roger Jean-Baptiste Robert Wets,

Order-preserving functions: Applications to majorization and order

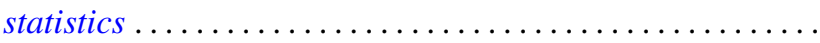

Wellington Ham Ow, An extremal length criterion for the parabolicity of

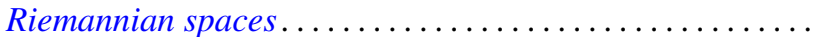

585

Wellington Ham Ow, Criteria for zero capacity of ideal boundary components of

Riemannian spaces...................................... 591

J. H. Reed, Inverse limits of indecomposable continua .................. 597

Joseph Gail Stampfli, Minimal range theorems for operators with thin spectra . . . 601

Roy Westwick, Transformations on tensor spaces..................... 613

Howard Henry Wicke, The regular open continuous images of complete metric

spaces ........................................... 621

Abraham Zaks, A note on semi-primary hereditary rings .............. 627

Thomas William Hungerford, Correction to: "A description of $\operatorname{Mult}_{i}\left(A^{1}, \cdots, A^{n}\right)$

by generators and relations" ............................. 629

Uppuluri V. Ramamohana Rao, Correction to: "On a stronger version of Wallis' formula".............................................. 629

Takesi Isiwata, Correction: "Mappings and spaces" ................... 630

Henry B. Mann, Josephine Mitchell and Lowell Schoenfeld, Correction to:

"Properties of differential forms in $n$ real variables" .... . .

James Calvert, Correction to: "An integral inequality with applications to the

Dirichlet problem"............................. 